\title{
Crescimento e qualidade de mudas de Citharexylum myrianthum em resposta à fertilização nitrogenada
}

\author{
Maria do Carmo Oliveira da Cunha Fernandes ${ }^{1 *}$ Eliane Cristina Sampaio de Freitas ${ }^{2}$ Haroldo Nogueira de Paiva ${ }^{1}$ \\ Silvio Nolasco de Oliveira Neto ${ }^{1}$
}

${ }^{1}$ Universidade Federal de Viçosa, Viçosa, Minas Gerais

${ }^{2}$ Universidade Federal Rural de Pernambuco, Recife, Pernambuco

*Author for correspondence: mariaducarmo28@ hotmail.com

Received: April 2018 / Accepted: December 2018 / Published: March 2019

\section{Resumo}

Citharexylum myrianthum, espécie nativa da Mata Atlântica, tem sido muito utilizada em programas de recuperação de matas ciliares. Entretanto, não existem trabalhos sobre a resposta dessa espécie à fertilização com nitrogênio, nutriente absorvido em maior quantidade pelas plantas. Nesse sentido, o objetivo desse trabalho foi avaliar o crescimento inicial e a qualidade de mudas de Citharexylum myrianthum em resposta à adubação nitrogenada. Foram testadas seis doses de $\mathrm{N}\left(0,80,160,240,320\right.$ e $\left.400 \mathrm{mg} \mathrm{dm}^{-3}\right)$, utilizando como fonte o sulfato de amônio. Aos 145 dias após a semeadura, foi realizada a medição da altura da parte aérea $(\mathrm{H})$ e do diâmetro do coleto (DC), e obtidos o peso de matéria seca da parte aérea (MSPA), das raízes (MSR) e total (MST). Também foram calculadas as relações: H/DC, H/MSPA, MSPA/MSR e o Índice de Qualidade de Dickson (IQD). A fertilização nitrogenada possibilitou ganhos significativos $(p<0,01)$ no crescimento e na qualidade de mudas de Citharexylum myrianthum. Não foi possível estimar a dose ótima de nitrogênio para a produção de mudas dessa espécie, pois essa irá ocorrer em doses maiores que as estudadas.

Palavras-chave: Nutrição mineral de plantas; Pau-viola; Produção de mudas.

\begin{abstract}
Citharexylum myrianthum, native species of the Atlantic Forest, has been widely used in recovery projects of riparian forests. However, there are no studies about the response of this specie to nitrogen fertilization, and that nutrient is the most absorbed by plants. Thus, this study aimed to evaluate the initial growth and quality of Citharexylum myrianthum seedlings in response to nitrogen fertilization. The nitrogen source used was ammonium sulfate and six $\mathrm{N}$ doses were tested $\left(0,80,160,240,320\right.$ and $\left.400 \mathrm{mg} \mathrm{dm}^{-3}\right)$. After 145 days of sowing, the height $(\mathrm{H})$, collar diameter $(\mathrm{CD})$, shoot dry matter weight (MSPA), root dry matter weight (MSR), total dry matter weight (MST) were obtained and the ratios of H/MSPA, H/DC, MSPA/MSR and the Dickson Quality Index (DQI) were calculated. Nitrogen fertilization resulted in significant gains $(\mathrm{p}<0.01)$ in growth and quality of Citharexylum myrianthum seedlings. It was not possible to estimate the nitrogen optimum dose for Citharexylum myrianthum seedling production, since that will occur at higher doses than those studied.
\end{abstract}

Keywords: Plants nutrition; Pau-viola; Seedling production.

\section{Introdução}

Uma das fases mais importantes para estabelecer povoamentos florestais com espécies nativas é a produção de mudas em quantidade e qualidade (Gonçalves et al. 2000). Mudas que apresentam boa qualidade possuem maiores taxas de crescimento e sobrevivência após o plantio em campo (Cruz et al. 2006), diminuindo os gastos com tratos culturais e replantio (Carneiro 1995).
Na determinação da qualidade das mudas são utilizados aspectos fenotípicos (morfológicos) ou fisiológicos, sendo que as características morfológicas são as mais utilizadas, devido à compreensão mais intuitiva por parte dos viveiristas (Gomes et al. 2002). Segundo Gonçalves et al. (2000), uma muda de boa qualidade deve ser vigorosa, estar bem nutrida e rustificada, e apresentar sistema radicular bem desenvolvido, sem enovelamentos.

Entre os fatores que afetam a qualidade das mudas podese citar o recipiente, substrato, qualidade das sementes, fertilização e o manejo das mudas no viveiro (Cruz et al. 2006). Segundo Tucci et al. (2009), um dos fatores responsáveis por perdas de mudas e elevada mortalidade das plantas após o plantio em campo é a fertilização inadequada do substrato. Assim, torna-se importante o uso de substrato apropriado com a quantidade de nutrientes necessária à demanda da espécie utilizada.

O nitrogênio é o nutriente absorvido em maiores concentrações pelas plantas, participando de diversas reações metabólicas (Cantarella 2007). Sua deficiência causa redução no crescimento das plantas, e um dos principais sintomas é o amarelecimento das folhas, inicialmente nas mais velhas, como resultado da proteólise (Davide e Faria 2008).

$\mathrm{O}$ fato do nitrogênio ser encontrado em vários estados de oxidação, com uma complexa dinâmica no solo, faz com que o manejo correto da fertilização nitrogenada seja um dos mais difíceis (Epstein e Bloom 2005). No solo o nitrogênio é encontrado sob a forma orgânica e inorgânica, sendo a maior parte na forma orgânica, como proteínas, peptídeos, quitina, ácidos nucléicos, bases nitrogenadas e ureia (Moreira e Siqueira, 2006). As formas inorgânicas são as que as plantas mais absorvem, sendo as principais a forma amoniacal $\left(\mathrm{NH}_{4}^{+}\right)$ e nítrica $\left(\mathrm{NO}_{3}{ }^{-}\right)$(Williams e Miller 2001). Em relação à preferência pela forma nítrica ou amoniacal, muitos trabalhos apresentam a preferência das espécies arbóreas pelo amônio. Segundo Grespan et al. (1998), a absorção de amônio demanda menor gasto de energia metabólica, quando comparada à de nitrato, uma vez que, para a absorção deste, é desnecessária a ação da nitrato redutase nas raízes.

Existe uma grande diversidade de espécies arbóreas nativas e cada uma tem exigências nutricionais específicas. No entanto, alguns viveiros adotam a mesma fertilização para todas as espécies, pois as operações diferenciadas ocasionam maior custo final das mudas (Davide e Silva 2008). As recomendações de nutrientes têm sido as que garantem o suprimento destes para as espécies mais exigentes, assim aquelas que possuem menor demanda são atendidas (Cruz et al. 2006). Contudo, o excesso de nutrientes pode provocar redução na qualidade das mudas, podendo prejudicar o crescimento das mesmas, além do gasto desnecessário de insumos, o que pode acarretar em prejuízos ambientais e financeiros.

Citharexylum myrianthum Cham., conhecida popularmente como pau-viola, é uma espécie pioneira da 
família Verbenaceae, nativa da Mata Atlântica, que pode alcançar até 20 metros de altura e 40 a $60 \mathrm{~cm}$ de diâmetro (Lorenzi 2002). Segundo esse autor, essa espécie tem preferência por ambientes úmidos a brejosos, tendo grande importância em projetos de recuperação de áreas ciliares degradadas.

Assim, tendo em vista a importância da Citharexylum myrianthum e a falta de estudos em relação à fertilização nitrogenada para essa espécie, o objetivo do presente trabalho foi avaliar o crescimento inicial e a qualidade de mudas de pau-viola em resposta às doses de nitrogênio.

\section{Material e Métodos}

O presente trabalho foi desenvolvido no Viveiro de Pesquisas Florestais do Departamento de Engenharia Florestal da Universidade Federal de Viçosa, MG, no período de setembro de 2016 a janeiro de 2017. Nesse período a temperatura média foi de $22{ }^{\circ} \mathrm{C}$ e a precipitação total de 624 mm (Estação Meteorológica de Viçosa, 2018). Segundo a classificação de Koppen, o clima da região é do tipo Cwb, mesotérmico, com verão chuvoso e inverno seco e frio.

As sementes de Citharexylum myrianthum (pau-viola) foram doadas pela Sociedade de Investigações Florestais (SIF). Para a quebra de dormência, as sementes foram deixadas imersas em água por 48 horas. A semeadura foi feita diretamente no recipiente, um tubete de plástico rígido de 55 $\mathrm{cm}^{3}$ de capacidade, utilizando substrato comercial, constituído de casca de pinus, vermiculita, carvão e turfa. Após a semeadura (duas sementes por recipiente), os tubetes foram deixados a pleno sol em bandejas suspensas cerca de 1 metro do solo. Aos 60 dias após a semeadura, quando as mudas apresentavam dois pares de folhas, foi realizado o raleio deixando a muda mais vigorosa. As irrigações foram feitas em intervalos de uma hora, sendo realizadas oito irrigações diárias (8:00 às 15:00).

Foram testadas seis doses de nitrogênio $(0,80,160,240$, 320 e $400 \mathrm{mg} \mathrm{dm}^{-3}$ ), tendo como fonte o sulfato de amônio, parceladas em 10 aplicações com intervalo de 7 dias após o raleio. A dose de nitrogênio foi baseada em Gonçalves et al. (2000), e a partir da dose recomendada (200 $\left.\mathrm{mg} \mathrm{dm}^{-3} \mathrm{de} \mathrm{N}\right)$ foram estipuladas doses acima e abaixo desse valor. $\mathrm{O}$ delineamento experimental foi o em blocos ao acaso (DBC) com 4 repetições, e parcela constituída por 25 plantas, resultando em um total de 600 plantas.

A adubação de base, incorporada ao substrato antes do preenchimento dos tubetes, foi feita com superfosfato simples na dose de $100 \mathrm{mg} \mathrm{dm}^{-3}$ de $\mathrm{P}_{2} \mathrm{O}_{5}$. E a adubação de cobertura foi realizada com cloreto de potássio na dose de $150 \mathrm{mg} \mathrm{dm}^{-3}$ de $\mathrm{K}_{2} \mathrm{O}$, parcelada em 6 aplicações intercaladas em 14 dias, tendo início após o raleio das mudas.

A medição da altura $(\mathrm{H})$ e do diâmetro do coleto (DC) das mudas foi feita aos 145 dias após a semeadura, utilizando régua e paquímetro digital, respectivamente. Posteriormente, foram realizadas a separação da parte aérea e da raiz e a lavagem do sistema radicular com auxílio de uma peneira de malha fina. As partes aéreas e as raízes das mudas foram colocadas em estufa a temperatura de $60{ }^{\circ} \mathrm{C}$ por quatro dias e depois foram pesadas em balança de precisão de $0.01 \mathrm{~g}$, obtendo-se o peso de matéria seca da parte aérea (MSPA) e das raízes (MSR). A soma do MSPA com MSR resultou no peso de matéria seca total (MST).

Além dessas características morfológicas, foram calculados os seguintes índices de qualidade de mudas: relação entre a altura da parte aérea e o diâmetro do coleto (H/DC), altura da parte aérea e o peso de matéria seca da parte aérea (H/MSPA), peso de matéria seca da parte aérea e peso de matéria seca das raízes (MSPA/MSR) e Índice de Qualidade de Dickson (IQD), em que:

$$
I Q D=M S T(H \div D C+M S P A \div M S R)
$$

Os dados obtidos foram interpretados estatisticamente por meio da análise de variância e equações de regressão. Com a finalidade de verificar se a distribuição dos erros é normal, foi aplicado o teste de normalidade (Teste de Lilliefors), a $5 \%$ de probabilidade. Para a escolha das equações de regressão foi considerada a significância dos coeficientes da regressão, o coeficiente de determinação $\left(\mathrm{R}^{2}\right)$ e também o realismo biológico.

\section{Resultados}

A fertilização nitrogenada resultou em efeitos significativos $(p<0,01)$ no crescimento das mudas de Citharexylum myrianthum para todas as características morfológicas e índices de qualidade de mudas avaliados, exceto para a relação H/DC (Tabela 1; Figura 1). Para o teste de normalidade não houve efeito significativo para nenhuma das características estudadas, exceto para a relação H/MSPA. $\mathrm{O}$ alto coeficiente de variação para algumas características morfológicas e alguns índices pode ser explicado pela utilização da propagação sexuada, alta variabilidade genética, e pela espécie estudada não ser domesticada.

Tabela 1. Resumo da análise de variância para as características morfológicas e para os índices de qualidade de mudas de Citharexylum myrianthum, avaliados aos 145 dias após a semeadura.

\begin{tabular}{lllllllllll}
\hline \multicolumn{10}{c}{$p$-valor $($ teste F) } \\
\hline FV & GL & H $(\mathbf{c m})$ & DC $(\mathbf{m m})$ & MSPA $(\mathbf{g})$ & MSR $(\mathbf{g})$ & MST $(\mathbf{g})$ & H/DC & H/MSPA & MSPA/MSR & IQD \\
\hline Bloco & 3 & 0,7871 & 0,0903 & 0,6444 & 0,9260 & 0,8178 & 0,6158 & 0,5216 & 0,8190 & 0,8347 \\
\hline N & 5 & 0,0000 & 0,0000 & 0,0000 & 0,0000 & 0,0000 & 0,0606 & 0,0000 & 0,0000 & 0,0000 \\
\hline CV $(\%)$ & & 38,37 & 35,42 & 68,73 & 60,80 & 65,07 & 6,75 & 88,95 & 19,38 & 60,34 \\
\hline
\end{tabular}

H - altura da parte aérea, DC - diâmetro do coleto, MSPA - peso de matéria seca da parte aérea, MSR - peso de matéria seca das raízes, MST- peso de matéria seca total, H/DC - relação da altura da parte aérea e diâmetro do coleto, H/MSPA - relação da altura da parte aérea e peso de matéria seca da parte aérea, MSPA/MSR - relação peso de matéria seca da parte aérea e peso de matéria seca das raízes e o IQD - Índice de Qualidade de Dickson. 


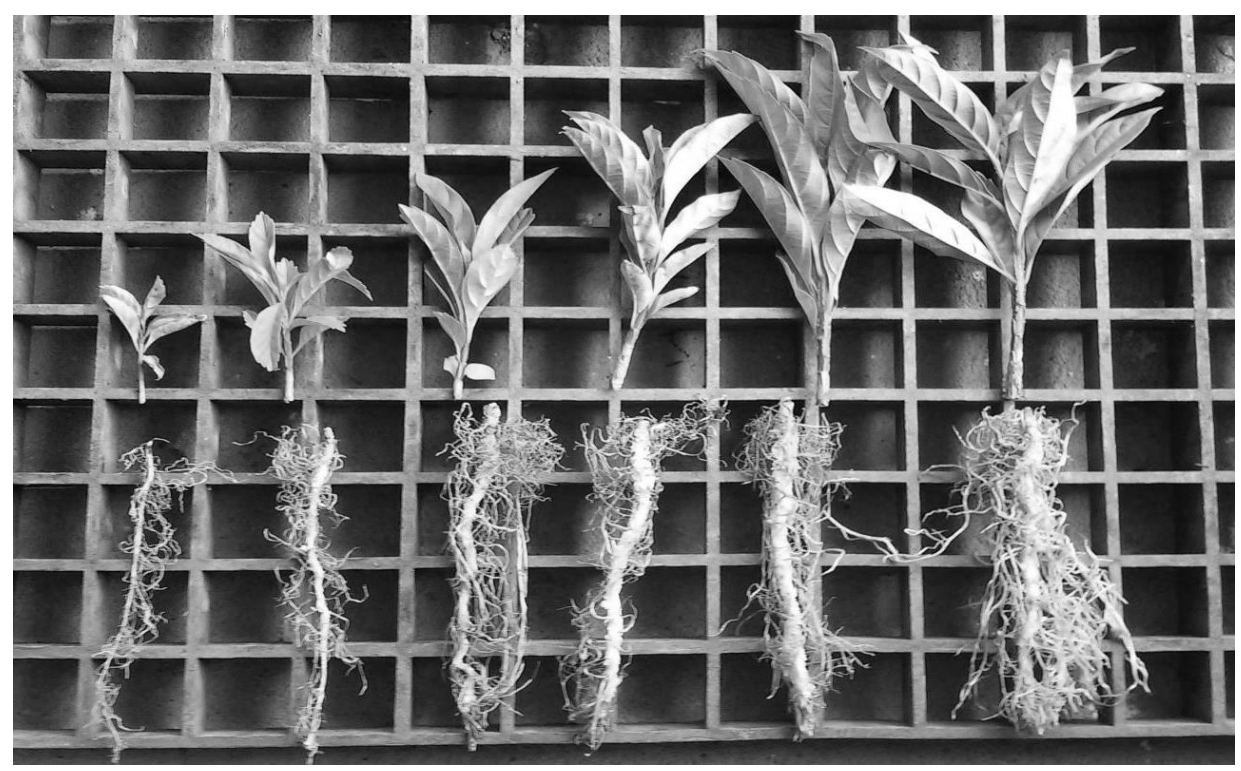

Figura 1 - Parte aérea e raiz de mudas de Citharexylum myrianthum, aos 145 dias após a semeadura. Da esquerda para a direita: doses de 0, 80, 160, 240, 320 e $400 \mathrm{mg} \mathrm{dm}^{-3} \mathrm{de} \mathrm{N}$ (Fonte: Arquivo pessoal).

Para todas as características morfológicas e IQD, a resposta ao aumento das doses de $\mathrm{N}$ foi linear positiva (Figura 2). Na maior dose testada $\left(400 \mathrm{mg} \mathrm{dm}^{-3}\right.$ ) foram obtidos 11,91 $\mathrm{cm} \mathrm{de} \mathrm{H} ; 4,81 \mathrm{~mm}$ de DC; 32,93 g, 25,70 g e 58,63 g de MSPA, MSR e MST,

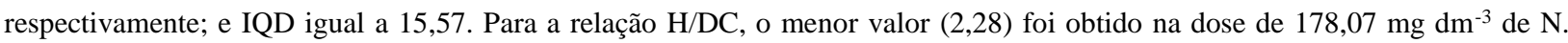
Apesar do teste de normalidade ter dado resposta significativa para a relação H/MSPA, os dados mostraram tendência exponencial e assim foi ajustado um modelo exponencial para esse índice. A melhor dose foi de $400 \mathrm{mg} \mathrm{dm}^{-3} \mathrm{de} \mathrm{N}$, resultando numa H/MSPA igual a 0,075 . Dose próxima $\left(360,75 \mathrm{mg} \mathrm{dm}^{-3} \mathrm{de} \mathrm{N}\right)$ foi encontrada para o maior valor da relação MSPA/MSR $(1,26)$.

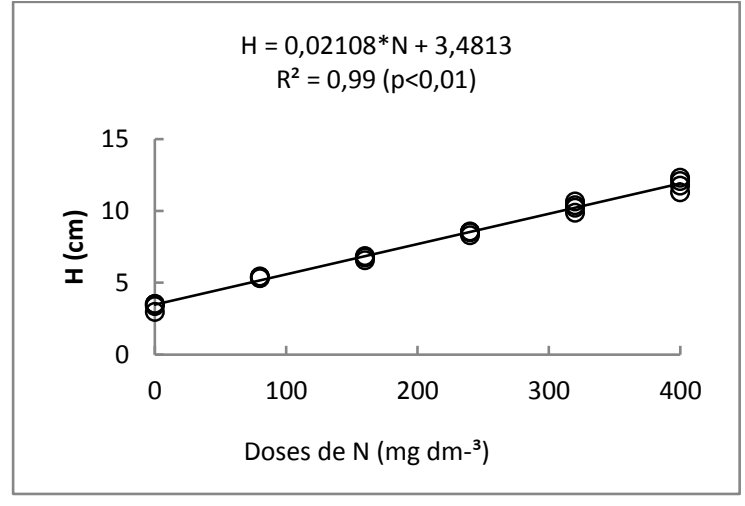

(a)

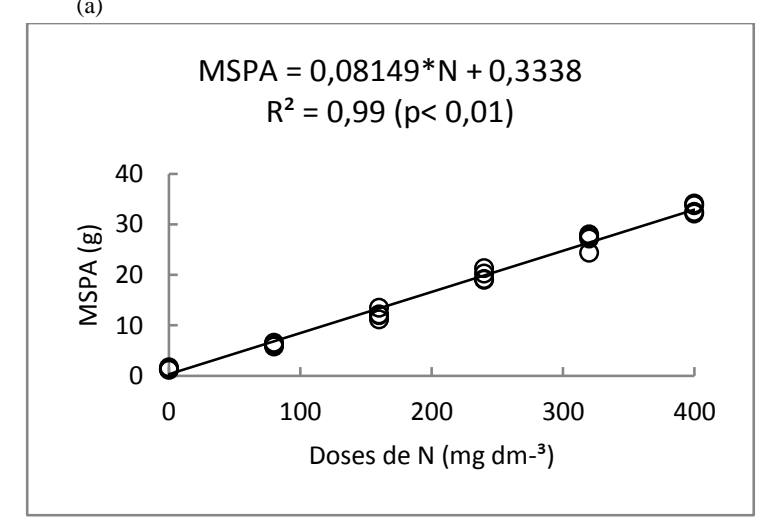

(c)
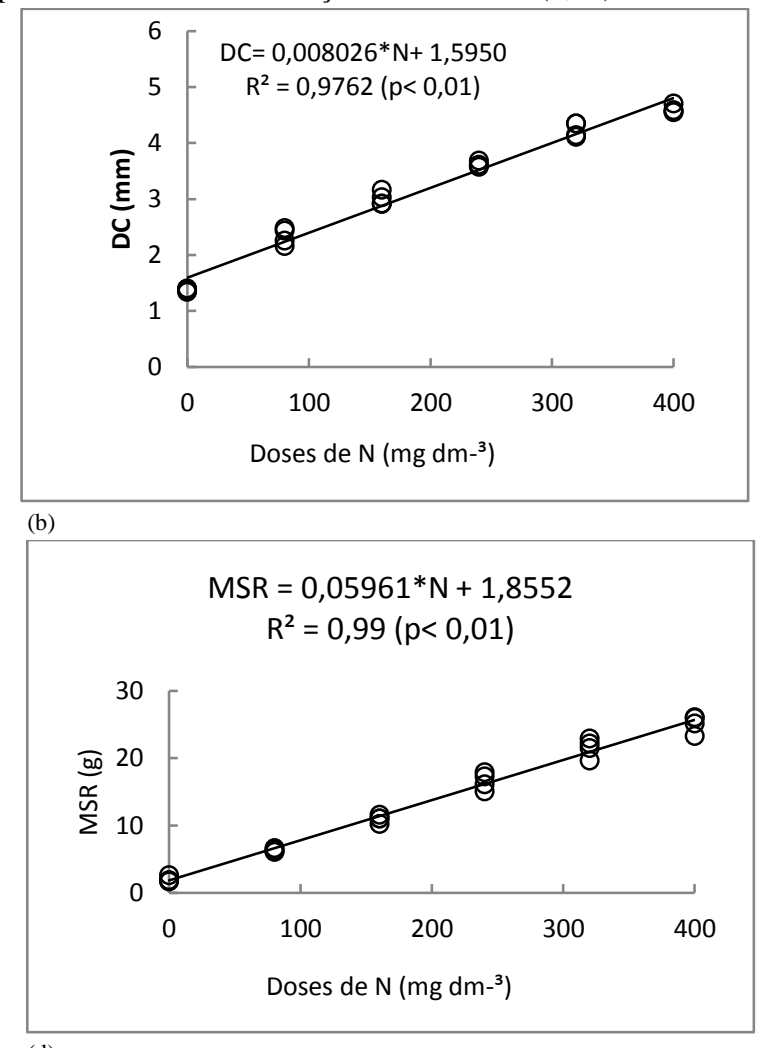

(d) 


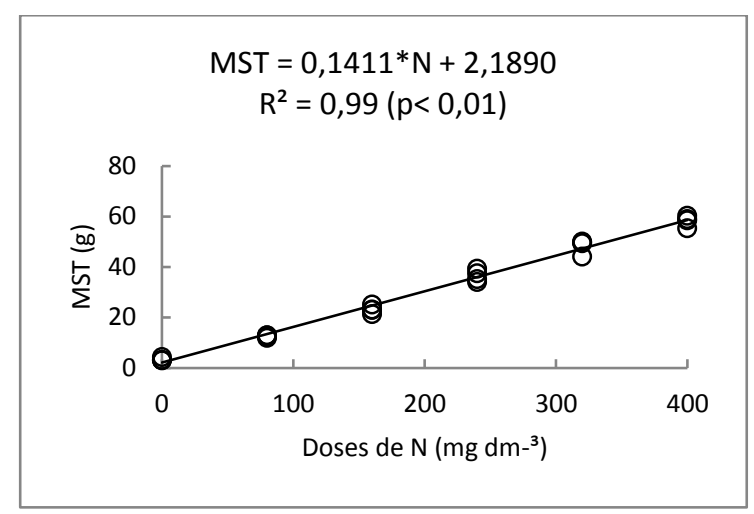

(e)

$$
\begin{gathered}
H / M S P A=2,2878 \operatorname{Exp}(-0,008556 * N) \\
R^{2}=0,91(p<0,05)
\end{gathered}
$$

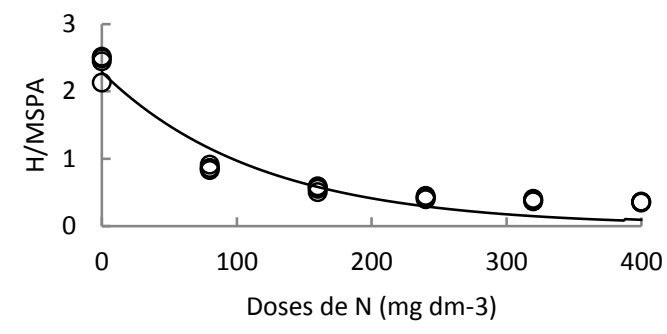

(g)

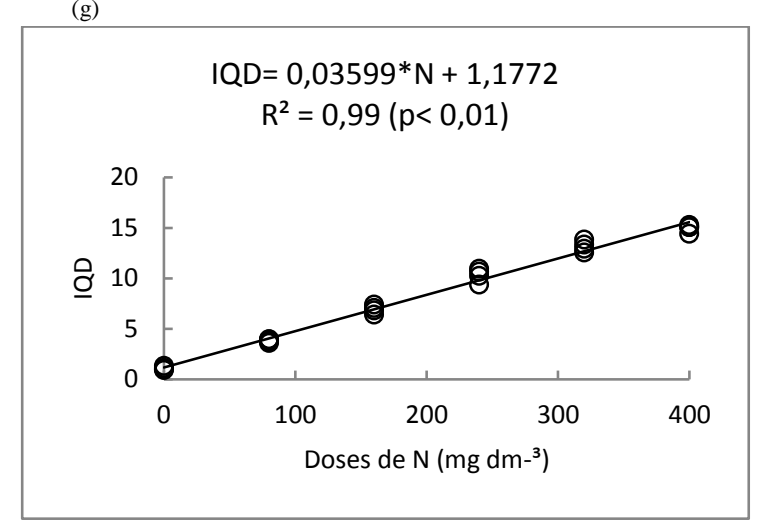

*Significativo a $1 \%$ pelo teste $\mathrm{T}$.

Figura 2 - (a) Altura da parte aérea (H), (b) diâmetro do coleto (DC), (c) peso de matéria seca da parte aérea (MSPA), (d) peso de matéria seca de raízes (MSR) e (e) peso de matéria seca total (MST), (f) relação altura/diâmetro do coleto (H/DC), (g) altura/peso de matéria seca da parte aérea (H/MSPA), (h) peso de matéria seca da parte aérea/peso de matéria seca de raízes (MSPA/MSR) e (i) Índice de Qualidade de Dickson (IQD) de mudas de Citharexylum myrianthum, em resposta à adubação nitrogenada, aos 145 dias após a semeadura.

\section{Discussão}

Diversos trabalhos com espécies arbóreas corroboram com o resultado obtido nesse estudo, em que a fertilização nitrogenada influenciou de forma positiva o crescimento inicial e a qualidade das mudas de espécies florestais nativas (Cruz et al. 2006, Gonçalves et al. 2008, Marques et al. 2009, Tucci et al. 2009, Gonçalves et al. 2010, Feitosa et al. 2011, Cruz et al. 2011, Caione et al. 2012, Ciriello et al. 2014, Mews et al. 2015, Goulart et al. 2016, Carvalho et al. 2016, Soares et al. 2017). O nitrogênio faz parte de vários compostos nas plantas, como aminoácidos, ácidos nucléicos e clorofila (Cantarella 2007), o que justifica sua absorção em maiores concentrações pelas plantas. Carnevali et al. (2016) avaliaram a eficiência nutricional de mudas de Stryphnodendron polyphyllum e observaram que o $\mathrm{N}$ e o $\mathrm{Ca}$ foram os nutrientes mais acumulados nos tecidos da planta.

Segundo Gonçalves et al. (2000), espécies dos estágios iniciais de sucessão têm maiores taxas de crescimento e,

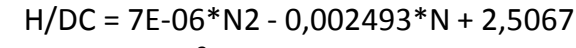

$R^{2}=0,57(p<0,01)$

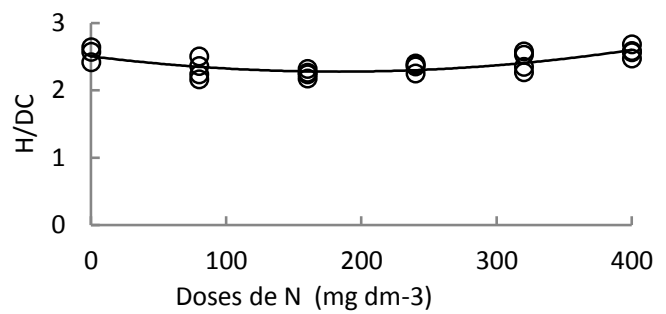

(f)

MSPA/MSR $=-4 \mathrm{E}-06 * \mathrm{~N} 2+0,002886 * \mathrm{~N}+$

0,7362

$R^{2}=0,93(p<0,01)$

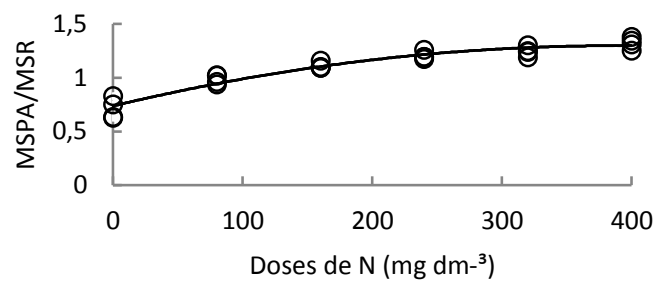

(h) consequentemente, maiores demandas por nutrientes e capacidade de absorção. Por esse motivo, esses autores ressaltam que espécies pioneiras e secundárias iniciais necessitam receber recomendações de fertilizantes de forma mais criteriosa, pois os riscos de terem o crescimento inicial prejudicado pela falta ou desbalanço de nutrientes são maiores que nas espécies das demais classes sucessionais. Entretanto, algumas espécies, mesmo que classificadas como pioneiras, não respondem à adubação nitrogenada no início do crescimento. Cardoso et al. (2016) avaliaram o efeito do nitrogênio no crescimento de mudas de sumaúma (Ceiba pentandra) em um Latossolo Amarelo distrófico e observaram que o crescimento das plantas foi pouco influenciado pelo fornecimento de doses crescentes de $\mathrm{N}$, sugerindo baixa exigência nutricional da espécie na fase inicial de crescimento. $\mathrm{O}$ mesmo foi observado por Campos de Jesus et al. (2016) para mudas de Inga laurina, em que a ausência de fertilização suplementar com $\mathrm{N}$ favoreceu a nodulação para fixação biológica de N. Como pode ser visto, 
não se deve generalizar a resposta ou ausência dela em relação aos grupos ecológicos. A resposta à fertilização nitrogenada depende de inúmeros fatores, como ambiente, genótipo, dose e fonte de nitrogênio (Marques et al. 2006).

A fertilização nitrogenada adequada promove incremento na altura das mudas das espécies florestais (Gomes e Paiva 2011). Efeito linear da aplicação de nitrogênio sobre a altura da parte aérea também foi verificado por Marques et al. (2006) para produção de mudas de Mimosa caesalpiniaefolia (sabiá), utilizando $200 \mathrm{mg} \mathrm{dm}^{-3}$ de $\mathrm{N}$ como a maior dose testada. A altura da parte aérea é uma das características mais importantes para avaliar o padrão de qualidade de mudas de espécies florestais, correlacionando-se positivamente com o crescimento no campo (Gomes e Paiva 2011). Essa característica morfológica é uma das mais utilizadas, por ser de fácil observação nos viveiros e não precisar destruir as mudas. Entretanto, a utilização dessa característica para comparar a qualidade das mudas é recomendada por Gomes e Paiva (2011) somente quando as mudas forem da mesma espécie e quando as técnicas de produção e as condições ambientais forem semelhantes.

O diâmetro do coleto também possui fácil mensuração e não é um método destrutivo. Apenas o diâmetro do coleto ou sua combinação com a altura representa uma das melhores características para avaliar o padrão de qualidade de mudas (Gomes e Paiva 2011). Segundo esses autores, o diâmetro do coleto chega a explicar 70 a $80 \%$ das diferenças que existem no peso de matéria seca das mudas. $\mathrm{O}$ valor do diâmetro do coleto encontrado nesse estudo é próximo ao recomendado (5 a $10 \mathrm{~mm}$ ) por Gonçalves et al. (2000) para mudas de boa qualidade. No entanto, segundo Gomes e Paiva (2011), não existe um valor que define o padrão de qualidade de mudas, pois isto depende da espécie, do local, dos métodos e das técnicas de produção.

A produção de biomassa é uma das melhores características para avaliar a qualidade das mudas, apesar de destrutiva, pois reflete a fotossíntese líquida da planta. Smarsi et al. (2011) observaram que com o aumento das doses de N, houve aumento linear do teor de clorofila nas folhas das mudas de Litchi chinensis (lichia). Já Cruz et al. (2006) observaram respostas quadráticas para essas características na produção de mudas de Samanea inopinata (sete-cascas).

Apesar de ser considerada uma das melhores características para avaliar a qualidade das mudas, a matéria seca é pouco utilizada em viveiros, por se tratar de um método destrutivo e necessitar de estufa e balança de precisão. $\mathrm{Na}$ maior dose de $\mathrm{N}$ testada, houve um aumento da matéria seca total de mais de $2000 \%$ em relação à testemunha.

A resposta ao $\mathrm{N}$ foi maior para a matéria seca da parte aérea (maior coeficiente angular da regressão) que para raiz. Leite et al. (2017) observaram que com o aumento da fertilização nitrogenada em mudas de Handroanthus impetiginosus, a planta investiu maior potencial de crescimento da parte aérea comparada com o sistema radicular, resultando em uma maior produção de folhas. $\mathrm{Na}$ fase inicial da produção de mudas é aplicada maior quantidade de nitrogênio, com o objetivo de aumentar a área foliar e, consequentemente, proporcionar maior atividade fotossintética (Silveira e Higashi 2003), o que resulta em maior incremento de matéria seca da parte aérea. A produção de raízes também tem grande influência na sobrevivência e no crescimento inicial das mudas no campo, sendo que quanto mais abundante for o sistema radicular, maior a sobrevivência (Gomes e Paiva 2011).

Apesar de existir inúmeros trabalhos mencionando a relação das características morfológicas com o crescimento e a sobrevivência das mudas em campo, a observação delas de forma isolada pode acarretar em interpretações errôneas, como por exemplo, mudas com maiores alturas ocasionadas pelo estiolamento, mas com pior qualidade (Fonseca et al. 2002).

A relação H/DC representa o equilíbrio de desenvolvimento das mudas (Carneiro 1995). Esse índice também é conhecido como quociente de robustez, e quanto menor o seu valor, melhor é a qualidade da muda e maior a capacidade de sobrevivência e estabelecimento após o plantio (Gomes e Paiva 2011).

Outro índice de qualidade de mudas é o H/MSPA, que, de acordo com Gomes e Paiva (2011), quanto menor o quociente obtido dessa relação, mais lenhificada será a muda e maior deverá ser sua sobrevivência no campo. Cruz et al. (2006) encontraram resposta quadrática para essa relação em mudas de Samanea inopinata (sete-cascas), encontrando índice médio de 0,86 .

Em relação à distribuição de biomassa nas mudas, Gomes e Paiva (2011) mencionam que o melhor resultado para a relação MSPA/MSR é igual a 2,0. Nesse estudo não foi obtida relação entre parte aérea e raiz igual a 2. Marques et al. (2009) encontraram o melhor índice $(2,17)$ na dose de $59,90 \mathrm{mg} \mathrm{dm}^{-3}$ de N para a produção de mudas de Piptadenia gonoacantha (jacaré) utilizando um Latossolo como substrato. Cabe ressaltar, que o maior ou menor crescimento da parte aérea em relação ao sistema radicular vai depender do genótipo e da disponibilidade de recursos de crescimento (água e nutrientes) (Gonçalves e Mello 2000). A utilização de doses de $\mathrm{N}$ superiores à requerida pela espécie cultivada pode causar o crescimento deficiente do sistema radicular e sua desproporcionalidade com a parte aérea, o que diminui a qualidade e a sobrevivência das mudas no campo (Gomes e Paiva 2011).

Entre os índices usados, o Índice de Qualidade de Dickson (IQD) é considerado um dos melhores. Segundo Fonseca et al. (2002), esse índice é um bom indicador da qualidade das mudas, pois inclui a robustez e o equilíbrio da distribuição da biomassa da muda no seu cálculo, e quanto maior o valor do índice, melhor será a qualidade da muda. Como a resposta do IQD para as doses de $\mathrm{N}$ foi linear, não foi possível encontrar a melhor dose de nitrogênio para esse índice. Cruz et al. (2006) encontraram resposta quadrática para esse índice em mudas de Samanea inopinata (setecascas), sendo que o maior valor foi de 36,49 para as adubações feitas com sulfato de amônio.

Apesar de ser possível determinar as doses ótimas para as relações citadas anteriormente, isso não é o suficiente para indicar uma dose de nitrogênio para a produção de mudas de Citharexylum myrianthum, já que todas as características morfológicas analisadas (H, DC, MSPA, MSR e MST) e o IQD apresentaram resposta linear positiva. No entanto, deve se ter cuidado com aplicações de $\mathrm{N}$ em quantidades excessivas, uma vez que essas podem gerar menor grau de rustificação das mudas, o que diminui a resistência das mesmas, principalmente no que diz respeito à seca (Gomes e Paiva 2011).

\section{Conclusões}

A aplicação de nitrogênio proporciona ganhos significativos no crescimento inicial de mudas de Citharexylum myrianthum.

A dose ótima de $\mathrm{N}$ para a produção de mudas de qualidade desta espécie está acima de $400 \mathrm{mg} \mathrm{dm}^{-3}$.

\section{Referências}

Caione G, Lange A, Schoninger EL (2012). Crescimento de mudas de Schizolobium amazonicum (Huber ex Ducke) em substrato fertilizado com nitrogênio, fósforo e potássio. Scientia Forestalis, 40(94): 213221. 
Campos de Jesus MF, Souza Júnior JO, Góes GS, BritoRocha E, Mielke MS (2016). Crescimento e qualidade de mudas de Inga laurina em função do substrato e adubação suplementar. Pesquisa Florestal Brasileira, 36(86): 53-159. doi: 10.4336/2016.pfb.36.86.917.

Cantarella H. Nitrogênio. In: Novais RF, Alvarez VVH, Barros NF, Fontes, RLF, Cantarutti, RB, Neves JCL. (Eds.) (2007). Fertilidade do solo. Viçosa-MG: SBCS, p. $376-449$.

Cardoso AAS, Santos JZL, Tucci, CAF, Silva Júnior CH, Venturin N (2016). Respostas nutricionais de mudas de sumaúma à adubação nitrogenada, fosfatada $\mathrm{e}$ potássica. Científica, 44 (3): 421-430. doi: 10.15361/1984-5529.2016v44n3p421-430

Carneiro, JGA (1995). Produção e controle de qualidade de mudas florestais. Curitiba: UFPR/FUPEF; Campos: UENF, 451p.

Carnevali NHS, Marchetti ME, Vieira MC, Carnevali TO, Ramos, DD (2016). Eficiência nutricional de mudas de Stryphnodendron polyphyllum em função de nitrogênio e fósforo. Ciência Florestal, 26(2): 449-461. doi: $10.5902 / 1980509822746$

Carvalho AO, Bergamin AC, Evaristo AP, Neves, AHB, Carmo CCA, Junior, JNDSG (2016). Initial growth of 'paricá' (Schizolobium amazonicum) seedlings under different nitrogen doses. Nativa , 4(2): 112-115. doi 10.14583/2318-7670.v04n02a11.

Ciriello V, Guerrini IA, Backes C (2014). Doses de nitrogênio no crescimento inicial e nutrição de plantas de guanandi. Cerne, 20(4): 653-660. doi: $10.1590 / 01047760201420041445$.

Cruz CAF, Paiva HN, Guerrero, CRA (2006). Efeito da adubação nitrogenada na produção de mudas de setecascas (Samanea inopinata (Harms) Ducke). Revista Árvore, 30(4): 537-546. doi: 10.1590/S010067622006000400006

Cruz CAF, Paiva HN, Cunha ACMCM, Neves, JCL (2011). Macronutrientes na produção de mudas de canafístula em Argissolo-Vermelho Amarelo da região da zona da mata, MG. Ciência Florestal, 21 (3): 445457.

Davide AC, Faria JMR (2008). Viveiros Florestais. In: Davide AC, Silva EAA. Produção de sementes e mudas de espécies florestais. Lavras: UFLA, p.83-124.

Davide AC, Silva EAA (2008). Produção de sementes e mudas de espécies florestais. Lavras: UFLA. 175p.

Epstein E, Bloom AJ (2005). Mineral nutrition of plants: principles and perspectives. 2. ed. Massachussets: Sinauer, $380 \mathrm{p}$.

Estação Meteorológica de Viçosa (2018). Dados Meteorológicos. Disponível em: http://www.posmet.ufv.br/?page_id=1253. Acesso: 24 de out. de 2018.

Feitosa DG, Maltoni KL, Cassiolato AMR, Paiano MO (2011). Crescimento de mudas de gonçalo-alves (Astronium fraxinifolium) sob diferentes fontes e doses de nitrogênio. Revista Árvore, 35(3): 401-411. doi: 10.1590/S0100-67622011000300004.

Fonseca EDP, Valéri SV, Miglioranza, E, Fonseca NAN, Couto L (2002). Padrão de qualidade de mudas de
Trema micranta (L.) Blume, produzidas sob diferentes períodos de sombreamento. Revista Árvore, 26(4): 515-523. doi: 10.1590/S0100-67622002000400015.

Gomes JM, Couto L, Leite HG, Xavier A, Ribeiro, SGL (2002). Parâmetros morfológicos na avaliação da qualidade de mudas de Eucalyptus grandis. Revista Árvore, 26(6): 655-664. doi: 10.1590/S010067622002000600002 .

Gomes JM, Paiva HN (2011). Viveiros florestais. Viçosa: Editora UFV, 116p. (Série Didática).

Gonçalves JDM, Santarelli EG, MORAES NETO SD Manara, MP (2000). Produção de mudas de espécies nativas: substrato, nutrição, sombreamento e fertilização. In: Gonçalves, J. L. de M.; Benedeti, V. (Eds.) Nutrição e fertilização florestal. Piracicaba: IPEF, p. 309-350.

Gonçalves, EO, Paiva HN, Neves JCL, Gomes JM (2008). Crescimento de mudas de angico-vermelho (Anadenanthera macrocarpa (Benth.) Brenan) sob diferentes doses de macronutrientes. Revista Árvore, 32(6): 1029-1040. doi: 10.1590/S010067622008000600008 .

Gonçalves EO, PAIVA HN, Neves JCL, Gomes JM (2010). Crescimento de mudas de sanção-do-campo (Mimosa caesalpiniaefolia Benth.) sob diferentes doses de macronutrientes. Scientia Forestalis, 38(88): 599-609.

Goulart, L. M. L. et al. Produção de mudas de Ipê-amarelo (Tabebuia serratifolia) em resposta a fertilização nitrogenada. Floresta e Ambiente, v. 24, p. 1-9, 2016. DOI: $10.1590 / 2179-8087.137315$.

Gonçalves, JLM; Mello, SLM. O sistema radicular das árvores. In: Gonçalves, JLM; Benedetti, V. Nutrição e fertilização florestal. Piracicaba: IPEF, 2000. p.218267.

Grespan SL, Dias LE, Novais RF (1998). Crescimento e parâmetros cinéticos de absorção de amônio e nitrato por mudas de Eucalyptus spp submetidas a diferentes relações amônio/nitrato na presença e ausência de fósforo. Revista Brasileira de Ciências do Solo, 22: 667-674. doi: 10.1590/S0100-06831998000400012.

Leite MS, Freitas RMO, Leite TS, Dombroski JLD, Santos Junior JH (2017). Growth and morphological responses of Handroanthus impetiginosus (Mart. ex DC.) Mattos seedlings to nitrogen fertilization. Bioscience Journal, 33(1): 88-94. doi: 10.14393/BJv33n1a2017-32736.

Lorenzi H (2002). Árvores brasileiras: manual de identificação e cultivo de plantas arbóreas nativas do Brasil. 4. ed. Nova Odessa, SP: Instituto Plantarum, v.1, 384p.

Marques VB, Paiva HN, Gomes JM, Neves JCL (2006). Efeitos de fontes e doses de nitrogênio no crescimento de mudas de sabiá (Mimosa caesalpiniaefolia Benth.) Scientia Forestalis, (71): 77-85.

Marques LS, Paiva HN, Neves JCL, Gomes JM., Souza PHD (2009). Crescimento de mudas de jacaré (Piptadenia gonoacantha (Mart.) J.F. Macbr.) em diferentes tipos de solos e fontes e doses de nitrogênio. Revista Árvore, 33(1): 81-92. doi: 10.1590/S010067622009000100009 . 
Mews CL, Sousa JD, Azevedo GTOS, Souza AM (2015).

Efeito do hidrogel e ureia na produção de mudas de Handroanthus ochraceus (Cham.) Mattos. Floresta e Ambiente, 22(1): 107-116. doi: 10.1590/21798087.080814.

Moreira FMS, Siqueira JO (2006). Transformações bioquímicas e ciclos dos elementos no solo. In: Moreira FMS, Siqueira JO. Microbiologia e bioquímica do solo. 2. ed. Lavras, MG: Editora UFLA, p. 313-404.

Silveira RLVA, Higashi EN (2003). Fertirrigação na produção de mudas de Pinus. Addubare, (6): 1-4.

Smarsi RC, Oliveira GF, Reis LL, Chagas EA, Pio R, Mendonça V, Chagas PC, Curi PN (2011). Efeito da adubação nitrogenada na produção de mudas de lichieira. Revista Ceres, 58(1): 129-131. doi: 10.1590/S0034-737X2011000100020.

Soares CB, Freitas ECS, Paiva HN, Neves JCL (2017). Fontes e doses de nitrogênio no crescimento e qualidade de mudas de Cassia grandis e Peltophorum dubium. Revista Árvore, 41(2):1-9. doi: 10.1590/180690882017000200014

Tucci CAF, Lima HN, Lessa JF (2009). Adubação nitrogenada na produção de mudas de mogno (Swietenia macrophylla King). Acta Amazonica, 39(2): 289 -294. doi: 10.1590/S0044-59672009000200007.

Williams LE, Miller AJ (2001). Transporters responsible for the uptake and partitioning of nitrogenous solutes. Annual Review of Plant Physioloy and Pant Molecular Biology, 52:659-688. doi:

10.1146/annurev.arplant.52.1.659. 